\section{Elimination of \\ Dumbbell Bands and \\ Enhancement of \\ Resolution in MADGE \\ Using Delayed Start \\ Electrophoresis}

BioTechniques 28:32-34 (January 2000)

Microplate array diagonal gel electrophoresis (MADGE) is a highthroughput electrophoresis system that enables the simultaneous assay of many samples for known mutations $(1,2)$. Problems noted often in electrophoresis include the distortion of the bands into dumbbell shapes sometimes with streaking or skiing down the sides of the gel track (Reference 5 and Figure 1). Uneven heating of the gel can result in the distortion of the bands. Direct thermal control or low run voltage prevents this problem, which is caused by amperometric heating, but in our expe- rience, this has not resolved the dumbbelling of the bands.

Insertion or deletion polymorphism can be detected in acrylamide-derived gels through the formation of heteroduplexes, which have a considerably lower mobility in the gel when compared with homoduplexes (3). Heteroduplex generation by post-PCR annealing allows immediate genotyping by electrophoresis without further intervening steps. However, distortion of the bands together with the skiing effect can compromise the resolution of heteroduplexes and homoduplexes with a consequent risk of miscalling of genotypes (Figure 1). The fragments were visualized using Image Quant 5.0 on Fluorimager 595 (Molecular Dynamics, Sunnyvale, CA, USA) after an initial Vistra $^{\mathrm{TM}}$ Green nucleic acid stain (Amersham Life Science, Buckinghamshire, England) after the staining of the gels (20 $\mathrm{min})$ according to the manufacturer's instructions.

The idea of examining a delayed start electrophoresis came from the observation that, when using an 8-channel pipet for loading MADGE gels, the distortion of the bands was more severe in the last set loaded with little to no distortion in the first set. The samples at the top of the gel have a 5 min delay in the start of electrophoresis, time that must allow considerable ionic diffusion through the walls of the wells (noticeable from marker dye diffusion), thus reducing the sample to gel ionic imbalance. We assumed this to be the factor responsible for the dumbbell effects.

The solutions we considered for this type of problem were the time course for sample ions to gel diffusional exchange, changing the constitution of the gel and changing the sample composition before or after PCR. Of all the possibilities tested, one provided a quick and efficient solution to the problem. A 5-10 min delay in the start of electrophoresis was sufficient to allow optimal resolution of the bands without any loss of product (Figure 2). Changing the ionic composition strength of the loaded sample to match that of the gel was also examined (Figures 1 and 2 ). As the conditions for each PCR are optimized for PCR yield, changing the sample composition would preferably take place after rather than before PCR.
Before loading onto the gel, the sample is mixed with the formamide dye mixture: $98 \%$ deionized formamide, $10 \mathrm{mM}$ EDTA $\mathrm{pH}$ 8.0, 0.025\% (wt/vol) xylene cyanole FF, $0.02 \%$ (wt/vol) bromophenol blue (4). Several ratio combinations of the dye mixture with 10× TAE (where TAE gels were used) were tested (no TAE [Figure 1], $0.9 \times$ TAE, $1.8 \times$ TAE, 3.6× TAE final concentration in sample loaded [Figure 2]). TAE supplementation within a broad range, 0.9-3.6 TAE, was relevant. A combination of PCR product, dye mixture and 10× TAE in the ratio of $5 / 1.5 / 1.5(\mathrm{vol} / \mathrm{vol} / \mathrm{vol})$ was successful; $5 \mu \mathrm{L}$ were loaded per MADGE well $(2 \times 2 \times 2 \mathrm{~mm}$ wells have $8 \mu \mathrm{L}$ maximum volume). The PCR contained: $50 \mathrm{mM} \mathrm{KCl}, 10 \mathrm{mM}$ Tris $\mathrm{pH}$ $8.3,0.001 \%(\mathrm{wt} / \mathrm{vol})$ gelatin, $0.2 \mathrm{mM}$ each dNTP, $0.2 \mathrm{mM}$ of each PCR primer, $1 \mathrm{mM} \mathrm{MgCl} 2$, Taq DNA polymerase $(0.2 \mathrm{U} / 10 \mu \mathrm{L})$. TAE buffer $(10 \times)$ contains Tris-base $0.399 \mathrm{M}$, sodium acetate $(0.8 \mathrm{M}), 3 \mathrm{H}_{2} \mathrm{O}(0.8 \mathrm{M})$, EDTA- $\mathrm{Na}_{2} 2 \mathrm{H}_{2} \mathrm{O}(40 \mathrm{mM})$ and acetic acid to $\mathrm{pH}$ 7.2.

The loaded sample was then reasonably matched to the gel so that equilibration time was reduced. In effect, the 5 $\mu \mathrm{L}$ sample undergoes a dialysis through four $2 \mathrm{~mm}$ square walls of the cubic well (and to the overlying buffer, if set up

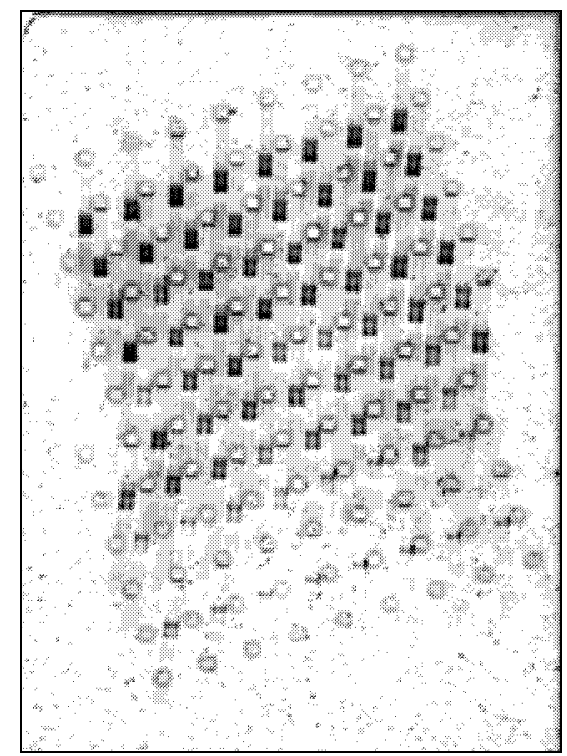

Figure 1. Artifacts that can occur with MADGE electrophoresis. Bands appear distorted into dumbbell shapes with skiing on either side of the track. 


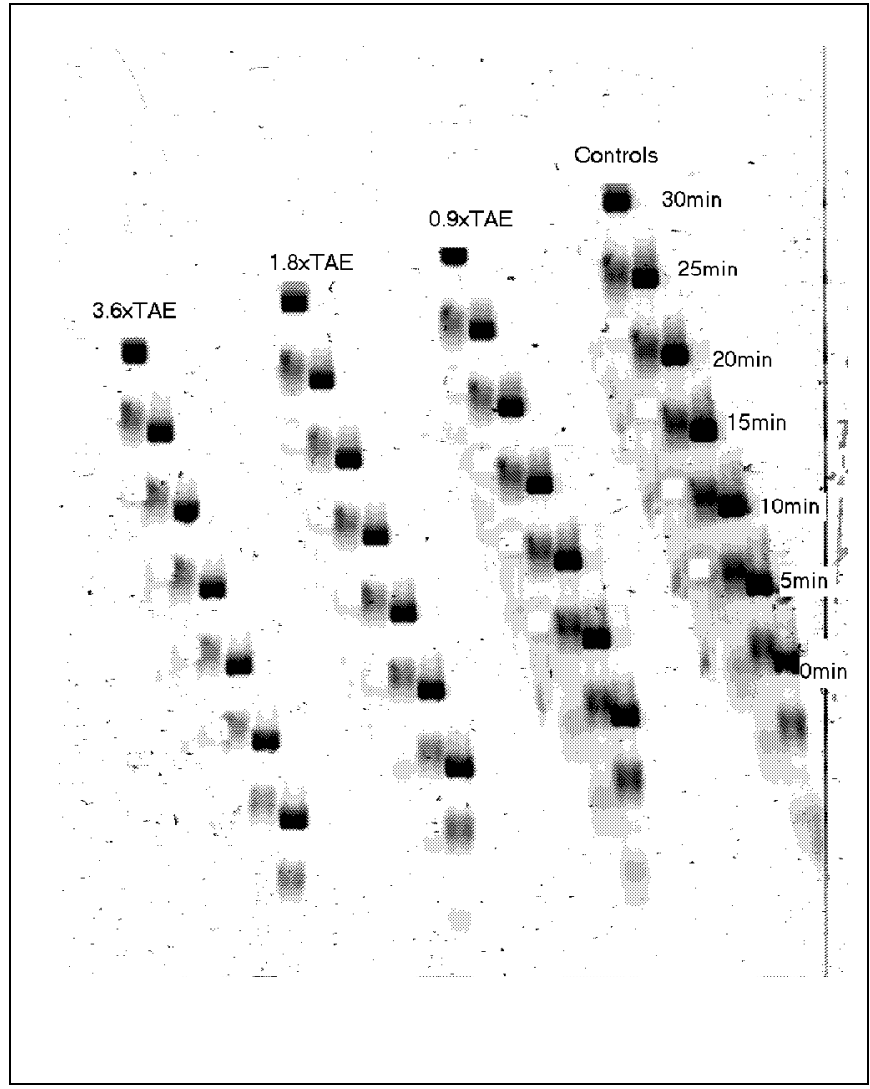

Figure 2. TAE titration and delayed start electrophoresis time course (delay time shown to right). Wells were loaded for each time point for each TAE concentration added (see text). The right-hand time increments refer to the bands on the gel.

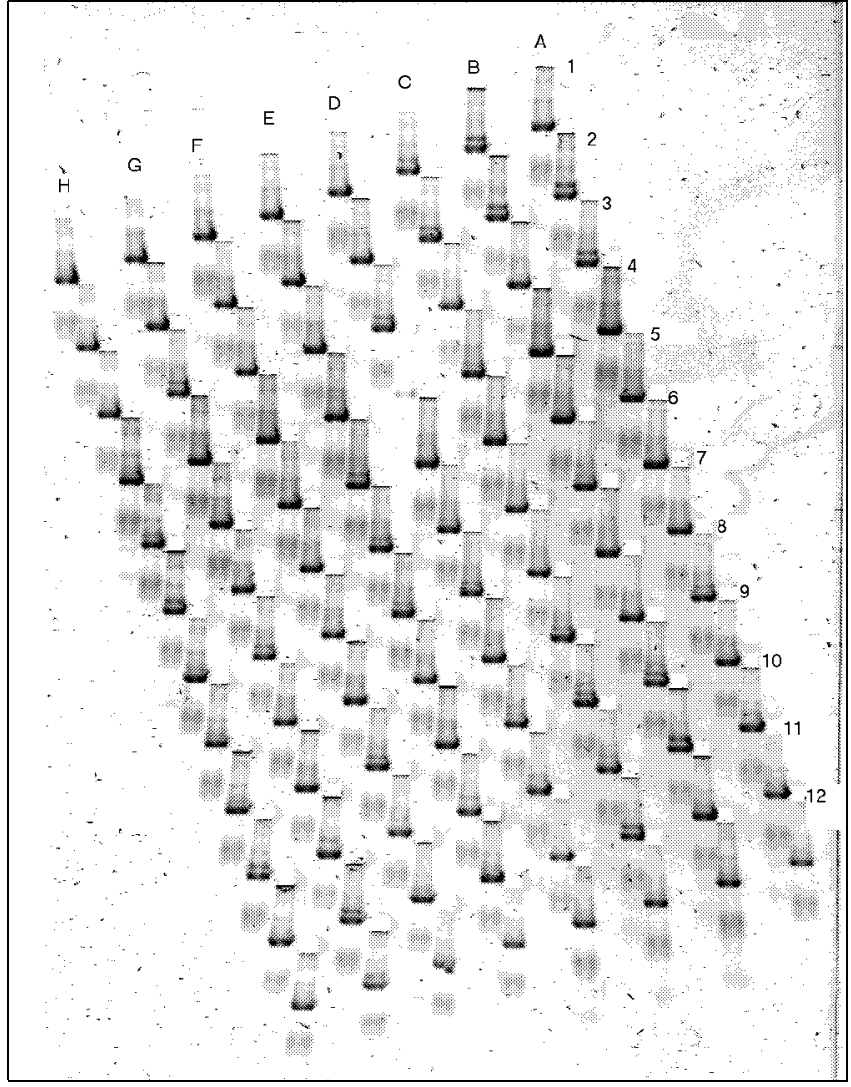

Figure 3. MADGE with delayed start electrophoresis. The bands are free of any distortion and the skiing effect has been eliminated. Tracks with heteroduplex bands are: B1, C1, A2, B2, C2, A3, D3, G3, E5, E6, H6, D7, E7, G7, C8, B9, C9, G9, B10, H10, C11, G11 and G12. submerged rather than with wicks or direct electrode contact to gel in a dry chamber) before the start of electrophoresis. Small distributed cubical wells such as those in MADGE arrays will benefit most from this approach.

Our results showed that a combination of the 5 min delay in the start of electrophoresis and attention to ionic constituents of sample loading buffer resolved the problem of dumbbell bands and of skiing along the sides of MADGE gel tracks (Figure 3). Sample preparation often receives much attention before electrophoresis (e.g., solid phase separations, extractions, and/or ethanol precipitations) (6). These approaches are labor intensive and difficult to automate.

The approach described-in effect, an in-gel-dialysis combined with approximate matching of sample to gel by titration of ions in the dye mix (Figure 2) - has given considerable improvements to the band quality (Figure 3 vs. Figure 1 ) and hence to the ease of data calling.

\section{REFERENCES}

1.Day, I. and S.E. Humphries. 1994. Electrophoresis for genotyping: microtitre array diagonal gel electrophoresis on horizontal polyacrylamide gels, hydrolink or agarose. Anal. Biochem. 222:389-395.

2.Day, I., S.E. Humphries, S. Richards, D. Norton and M. Reid. 1995. High-throughput genotyping using horizontal polyacrylamide gels with wells arranged for microplate array diagonal gel electrophoresis (MADGE). BioTechniques 19:830-835.

3.Keen, J., D. Lester, C. Inglehearn, A. Curtis and S. Bhattacharya. 1991. Rapid detection of single base mismatches as heteroduplexes on Hydrolink gels. Trends in Genetics 7:5.

4.Maniatis, T., E.F. Fritsch and J. Sambrook. 1982. Molecular cloning: A Laboratory Manual. CSH Laboratory Press, New York: Cold Spring Harbor $N Y$.

5.Patel, D. 1994. Gel electrophoresis: essential data. Wiley in association with BIOS Scientific, John Wiley and Son, NY.
6.Sealey, R. and E.M. Southern. 1982. Gel electrophoresis of DNA, p. 39-76. In D. Rickwood and B.D. Hames (Eds.), Gel electrophoresis of Nucleic Acids: A Practical Approach. IRL Press, Oxford, UK.

The MRC is thanked for support. A.M.V. is a Wessex Medical Trust PhD student. INMD is a Lister Institute Professor. Address correspondence to Anca M. Voropanov, Research Division of Human Genetics, (Mp808), School of Medicine, Southampton University, Southampton General Hospital, Southampton, England, UK. Internet: mamvl @ soton.ac.uk

Received 18 June 1999; accepted 14 October 1999

\section{Anca M. Voropanov and Ian \\ N.M. Day Southampton University Southampton General Hospital Southampton, England, UK}

